\title{
Transdermal fentanyl for the treatment of pain caused by osteoarthritis of the knee or hip: an open, multicentre study Xavier Le Loët ${ }^{1}$, Karel Pavelka ${ }^{2}$ and Ute Richarz*3
}

\author{
Address: ${ }^{1}$ Centre Hospitalier Universitaire de Rouen, Hôpitaux Rouen, Rouen, France, ${ }^{2}$ Institute of Rheumatology, Prague, Czech Republic and \\ JJanssen-Cilag Medical Affairs (Europe, Middle East \& Asia), Baar, Switzerland \\ Email: Xavier Le Loët - Xavier.Le-Loet@chu-rouen.fr; Karel Pavelka - pavelka@revma.cz; Ute Richarz* - urichar1@jacch.jnj.com \\ * Corresponding author
}

Published: 15 June 2005

BMC Musculoskeletal Disorders 2005, 6:31 doi:10.1 |86/|47|-2474-6-31

This article is available from: http://www.biomedcentral.com/l47/-2474/6/3

(C) 2005 Le Loët et al; licensee BioMed Central Ltd.

This is an Open Access article distributed under the terms of the Creative Commons Attribution License (http://creativecommons.org/licenses/by/2.0), which permits unrestricted use, distribution, and reproduction in any medium, provided the original work is properly cited.
Received: 20 October 2004

Accepted: 15 June 2005

\begin{abstract}
Background: This study was designed to evaluate the utility of transdermal fentanyl (TDF, Durogesic $\left.{ }^{\circledR}\right)$ for the treatment of pain due to osteoarthritis $(\mathrm{OA})$ of the knee or hip, which was not adequately controlled by non-opioid analgesics or weak opioids. The second part of the trial, investigating TDF in patients with rheumatoid arthritis (RA) is reported separately.
\end{abstract}

Methods: Current analgesia was optimised during a I-week run-in. Patients then received 28 days treatment with TDF starting at $25 \mu \mathrm{g} / \mathrm{hr}$, with the option to increase the dose until adequate pain control was achieved. Metoclopramide was taken during the first week and then as needed.

Results: Of the 159 patients recruited, 75 with OA knee and 44 with OA hip completed the treatment phase, 30 knee and 18 hip patients entered the one-week taper-off phase. The most frequently used maximum dose of TDF was $25 \mu \mathrm{g} / \mathrm{hr}$. The number of patients with adequate pain control increased during the run-in period from $4 \%$ to $27 \%$, and further increased during TDF treatment to $88 \%$ on day 28 . From baseline to endpoint, there were significant reductions in pain $(\mathrm{p}<0.00 \mathrm{I})$ and improvements in functioning $(\mathrm{p}<0.00 \mathrm{I})$ and physical $(\mathrm{p}<0.00 \mathrm{I})$ and mental $(\mathrm{p}<$ $0.05)$ health. Scores for 'pain right now' decreased significantly within 24 hours of starting TDF treatment. TDF was assessed favourably and $84 \%$ of patients would recommend it for OA-related pain. Nausea and vomiting were the most common adverse events (reported by $32 \%$ and $26 \%$ of patients respectively), despite prophylaxis with metoclopramide, which showed limited efficacy in this setting.

Conclusion: TDF significantly increased pain control, and improved functioning and quality of life. Metoclopramide appeared to be of limited value in preventing nausea and vomiting; more effective anti-emetic treatment may enable more people to benefit from strong opioids such as TDF. This study suggests that four weeks is a reasonable period to test the benefit of adding TDF to improve pain control in OA patients and that discontinuing therapy in cases of limited benefit creates no major obstacles.

\section{Background}

Osteoarthritis (OA) is a slowly developing articular dis- ease, characterized mainly by cartilage degeneration, which is reflected clinically by a gradual development of 
joint pain, stiffness, and loss of full range of movement. $\mathrm{OA}$ is the most common disease to affect synovial joints, being a major cause of musculoskeletal pain, reduced quality of life and disability. About $40-60 \%$ of patients with radiological osteoarthritic changes suffer from clinical symptoms of pain, stiffness and loss of mobility [1], and around $55 \%$ of patients with OA report pain as the worst aspect of the disease [2]. OA is strongly associated with ageing and, with an increasing elderly population, of major socioeconomic importance.

Current treatments for OA include a wide range of nonpharmacological, pharmacological and surgical options, although evidence to support their effectiveness is variable and there are no curative treatments. Therapies focus on reducing symptoms such as pain and stiffness, and minimalizing functional limitation and disability [3]. However, pain control is the primary aim of treating patients with $\mathrm{OA}$ and, in evaluating symptoms, pain should be the primary outcome variable [4]. Non-steroidal anti-inflammatory drugs play an important role in the pharmacological management of OA [5]. However, their lack of efficacy or potential toxicity may limit their use, in particular, the withdrawal of certain Cox-2 inhibitors has restricted the choice of therapies [3,5-7], and problems of persisting pain remain. Pain from OA may be caused by factors other than inflammation [8], therefore the logical next step in the treatment of OA-related pain is the use of strong opioids. Within a management programme aimed at improving physical and social function, guidelines recommend their use when other appropriate therapies have failed to provide adequate pain relief over a reasonable period of time [9-13].

Transdermal fentanyl (TDF), providing systemic delivery of fentanyl at a constant rate for 72 hours [14], has been shown to be effective in controlling pain and improving some quality of life parameters for people with chronic non-malignant pain [15-18]. The efficacy of opioids in controlling pain in patients with OA has been demonstrated in three randomized controlled trials [19-21]. Moreover, a prospective study to investigate the efficacy and tolerability of TDF in 243 patients with severe OA pain of the knee and/or hip demonstrated significant reductions in pain at rest and on movement, and provided evidence of functional improvement [22]. Very few patients needed doses higher than the $25 \mu \mathrm{g} / \mathrm{hr}$ starting dose after 30 days of treatment [22].

The present study was undertaken to evaluate the utility and safety of TDF for the treatment of pain associated with RA or with OA of the knee or hip, which was not adequately controlled by NSAIDs, Cox-2 inhibitors, paracetamol or weak opioids at optimal doses. As it was an openlabel study, it was not designed to prove efficacy of the treatment but to investigate practical aspects of opioid therapy, such as the usefulness of a test-period or concomitant use of antiemetic therapy and to serve as a pilot for a double-blind trial in this patient population. A test period for evaluation of a patient's response to opioid treatment is recommended [10] and in this study lasted 4 weeks. Results from the total study population and from patients with RA will be reported elsewhere. This paper reports the effects of TDF on pain and functioning in patients with OA of the knee or hip.

\section{Methods}

\section{Patient selection}

All participants were outpatients requiring supplementary analgesia because of moderate or severe pain, which was not adequately controlled with paracetamol, NSAIDs, Cox-2 inhibitors or weak opioids (e.g. tramadol or codeine). Because non-opioid analgesia is not always taken at sufficient doses to achieve pain control [24] the trial employed a run-in period during which analgesia was optimised. Patients who still had moderate or severe pain at the end of this period could enter the main treatment phase of the study. Patients had to be over 50 years old, have OA of the knee or hip and meet the OA criteria of the ACR [23]. They had to have radiographic evidence of OA, and be waiting for hip or knee replacement as indicated by an orthopaedic surgeon. If participants were taking corticosteroids and/or NSAIDs they had to have received a stable dose for at least three months before screening and expect to remain on a stable dose for the duration of the trial.

Patients were excluded from the study if they had received regular treatment with a strong opioid (e.g. morphine) or had received more than the maximum recommended dose of weak opioids or other analgesics in the four weeks before the study. Strong opioids other than fentanyl, supplementary weak opioids or other treatments that might alter the degree or nature of pain could not be started during the study. Patients were excluded if they had continuous pain of non-arthritis origin, or had undergone surgery/arthroscopy within 3 months, intra/peri articular injections for arthritis pain (e.g. steroid injection) within 6 weeks, or arthrocentesis within 4 weeks of the study start.

\section{Study design}

Screened patients satisfying the selection criteria each gave written informed consent before inclusion in this international, open, prospective trial. The study was carried out in accordance with the latest revision of the Declaration of Helsinki and Good Clinical Practice and was approved by independent local ethics committees. 
During the one-week run-in period, non-opioid analgesic treatment was increased to the maximum tolerated or maximum recommended dose, while weak opioids were kept stable. All patients with pain control rated as poor or very poor on a 5-point scale at the end of the run-in period started treatment for 28 days with transdermal fentanyl (TDF, Durogesic ${ }^{\circledast}$ ) at a dose of $25 \mu \mathrm{g} / \mathrm{h}$. Patches were replaced every 72 hours (3 days). Previous non-opioid analgesia was continued and kept stable, but weak opioids were discontinued. The dose of TDF could be increased in steps of $25 \mu \mathrm{g} / \mathrm{h}$ every 72 hours (days 3,6 and 9 ) if required (no maximum dose specified) until adequate pain control was achieved. After 28 days or if necessary for other reasons, e.g. if side effects occurred or the treatment was not effective, a similar downward titration regimen was employed. Paracetamol $500 \mathrm{mg}$ tablets were provided for supplementary analgesia and could be used in doses of up to $4 \mathrm{~g} /$ day. With the exception of paracetamol, other non-opioid analgesics were kept stable and no short acting opioids were added during down titration.

Metoclopramide $10 \mathrm{mg}$ three times a day was given to all patients for the prevention of nausea and vomiting during the first week of treatment, after which it was taken as needed.

\section{Assessments}

Patients were evaluated in the clinic at screening (day -7), at baseline (day 0), on days 7, 14 (optional because some clinicians see their patients only every 4 weeks) and on day 28 (trial end), on other days if dose adjustment was necessary, and at the end of a one or two week taperingoff period.

The primary efficacy variable was pain control, evaluated weekly on a five-point assessment scale ranging from very poor to excellent. For this assessment, the investigator presented the question "would you rate your pain control today as being excellent, good, moderate, poor or very poor?" Patients also completed a pain assessment questionnaire (shortened version of the Wisconsin Brief Pain Inventory (WBPI) [25]) (10-point rating scale: 0, best to 10 , worst). The degree of pain after 24 hours of treatment was assessed by asking patients about the amount of pain that they had 'right now'. Patients also recorded pain intensity in a diary on a five-point scale.

Patients completed a treatment assessment questionnaire consisting of 10 items scored on a Likert scale. The acute version of the SF-36 quality of life questionnaire [26] including eight quality of life domains (physical functioning, physical role limitations, emotional role limitations, social functioning, body pain, general mental health, vitality and general health perceptions) was completed after the run-in phase and at day 28. Functionality of
Table I: Patient characteristics

\begin{tabular}{lcc}
\hline & OA knee & OA hip \\
\hline N (baseline) & 102 & 57 \\
ITT analysis population & 91 & 52 \\
Mean age (years) \pm SE (range) & $68 \pm 0.9$ & $66 \pm 1.2$ \\
& $(49-88)$ & $(47-87)$ \\
Previous medication (\% of patients): & & \\
$\quad$ Non-opioids & 81 & 79 \\
Weak opioids & 50 & 40 \\
\hline
\end{tabular}

patients was assessed at the same time points using the Western Ontario and McMaster Universities Osteoarthritis Index LK 3.1 (WOMAC questionnaire) [27]. This has 24 questions that are each evaluated on a 5-point severity scale ( 0 , none to 4 , extreme), it assesses three areas: pain (5 questions), stiffness (2 questions) and functional impairment (17 questions). Maximum scores for each section differ and, therefore, scores are normalized by weighting severity to assist interpretation.

\section{Statistical analysis}

There was no formal sample size calculation. A safety analysis was performed using data from all patients entering the trial. An intent-to-treat analysis, comprising all OA patients with at least one post-baseline measurement of the primary endpoint (pain control) and treated at least once with trial medication, was also performed [28].

The ANCOVA model was used to analyse the change from baseline to endpoint, and the influence of baseline values. The ANCOVA model was also used to determine differences by centre. The Wilcoxon signed-rank test was used to compare intragroup results and results at each time point or endpoint with baseline, where applicable. Statistical tests were interpreted at the 5\% significance level (two-sided).

\section{Results \\ Patient characteristics}

A total of 159 patients, 102 with OA of the knee and 57 of the hip, were recruited into the study from 47 centres in 11 countries and started treatment with TDF. Patient characteristics are shown in Table 1. All but three patients used analgesic treatment in the month before screening and the treatments were similar for hip and knee groups. The most commonly used non-opioid analgesics were paracetamol $21 \%$, diclofenac $20 \%$ and rofecoxib $10 \%$. The most commonly used weak opioid was tramadol (33\%). During this time, half the patients (50\%) used a non-opioid only; $28 \%$ used a combination of a non-opioid and weak opioid and $18 \%$ a weak opioid only. 
Concomitant medication with possible analgesic effects during the treatment phase included paracetamol (69\%), NSAIDs (45\%), weak opioids (33\%), Cox-2 inhibitors $(13 \%)$, other analgesics $(8 \%)$, steroids $(6 \%)$, immunomodulating drugs $(1 \%)$, and strong opioids (1\%). Of those taking weak opioids, most took them on the first day of treatment only. Only two patients used opioid rescue medication and had to be considered as protocol violators. There was a reduction in the use of NSAIDs (from $45 \%$ to $18 \%$ ) and Cox-2 inhibitors (9\%) and paracetamol $(15 \%)$ after the first 24 hours of treatment. Rescue medication was required by $59 \%$ of participants, of whom almost all used non-opioids, particularly paracetamol. Most patients suffered from concomitant diseases: $59 \%$ of the patients had currently active cardiovascular diseases, $50 \%$ musculo-skeletal, $48 \%$ genito-urinary, $47 \%$ endocrine, and $19 \%$ gastrointestinal disease. The most frequently used non-analgesic concomitant therapies during treatment were metoclopramide $49 \%$ and omeprazole $12 \%$.

\section{Discontinuations}

Of the 159 participants recruited, 25\% withdrew from the trial during the treatment phase. Reasons for withdrawal were adverse events (35), insufficent response (1) and other reasons (4). Around half of the drop-outs (14\%, 21 of the 35 due to adverse events) occurred in the first week of TDF treatment (Figure 1). Of the 48 patients who started the optional tapering-off phase, 11 (23\%) discontinued prematurely (10 because of adverse events and one was lost to follow-up/surgery). Patients were treated for an average of $22.3 \pm 0.92$ days.

\section{Study medication}

All patients started at a dose of $25 \mu \mathrm{g}$ fentanyl/h and daily doses ranged from 25 to $125 \mu \mathrm{g} / \mathrm{h}$. The maximum dose was used by only one patient. The mean daily dose for week 1 was $26 \mu \mathrm{g} / \mathrm{h}$, which increased slightly to $37 \mu \mathrm{g} / \mathrm{h}$ in week 4 . Over half (54\%) of all patients used $25 \mu \mathrm{g} / \mathrm{h}$ as a maximum dose during the study (61\% OA knee, $42 \%$ OA hip).

\section{Evaluation of efficacy} Primary efficacy variable

Adequate pain control was defined as a score of 'moderate', 'good' or 'excellent' on the 5-point pain control assessment scale. The proportion of patients with adequate pain control increased during the one-week run-in period (during which doses of non-opioid analgesia were optimised) from $4 \%$ to $27 \%$. These patients continued in the study and accounted for the majority of protocol violators. At baseline, 25\% of patients reported very poor pain control, $48 \%$ poor and $25 \%$ moderate pain control, and there was no notable difference between those with OA knee and OA hip (Table 2). A further increase in the proportion of patients with adequate pain control was observed after TDF treatment, particularly in the first week of treatment (to $74 \%$ ) when $37 \%$ patients reported moderate, $29 \%$ good, and $8 \%$ excellent pain control. Adequate pain control was reported by $80 \%$ and $88 \%$ patients on days 14 and 28, respectively. At endpoint, 83\% of patients considered their pain controlled, with 37\% reporting moderate, 38\% good, and $8 \%$ excellent pain control (Table 2 \& Figure 2). About 10\% more patients with OA hip reported good or excellent pain control at endpoint than those with OA knee. However, of the patients who already experienced adequate pain control after the run-in phase (OA knee, 24 moderate and one good pain control; OA hip, 12 moderate and one good pain control), 50\% of both groups improved further during TDF treatment. Overall, $81 \%$ of participants with OA hip and $75 \%$ with OA knee improved from baseline to endpoint by at least one pain category.

\section{Effect size}

An effect size was calculated by assigning numerical values to the pain categories ( 1 = very poor, 2 = poor, $3=$ moderate, 4 = good, 5 = very good). This gives a mean pain control score at baseline of 2.03 (95\% CI 1.9, 2.2), rising to $3.33(3.2,3.5)$ at endpoint and a mean change from baseline of $1.3(\mathrm{SD} \pm 1.14)$. This gives an absolute effect size of 1.14 .

\section{Secondary efficacy variables \\ Pain control}

Patients reported a significant reduction in pain from baseline to endpoint for each WBPI item at every time point $(\mathrm{p}<0.001)$. The mean reduction in 'pain at its worst' was 1.8 points (from 8.1 to 6.3 ), 'pain at its least' was 1.6 points (from 4.4 to 2.8 ), and 'pain on average' was 2.0 points (from 6.4 to 4.4 ). The amount of trouble or bother the pain was causing also decreased by 2.7 points on average (from 7.2 to 4.5 ). The mean reduction in 'pain right now' was 2.6 points (from 6.1 to 3.5) from baseline to endpoint. A significant reduction in 'pain right now' was reported as early as 24 hours after baseline (1.3 points, from 6.0 to 4.7 ).

From patients' diaries, the mean score for degree of pain was significantly decreased at each time point, and from severe pain (score 3 ) to moderate pain (score 2 ) from the run-in period to endpoint $(\mathrm{p}<0.001)$. Results were similar for the patients' highest score for their degree of pain. Thus, while at baseline 58\% (79/137) reported severe/ extreme pain, 4\% (5) mild, and only two patients were without pain, by endpoint $41 \%(56 / 138)$ reported moderate pain, 30\% (41) mild and 7\% (9) no pain. There was little difference between the OA knee and OA hip group. 


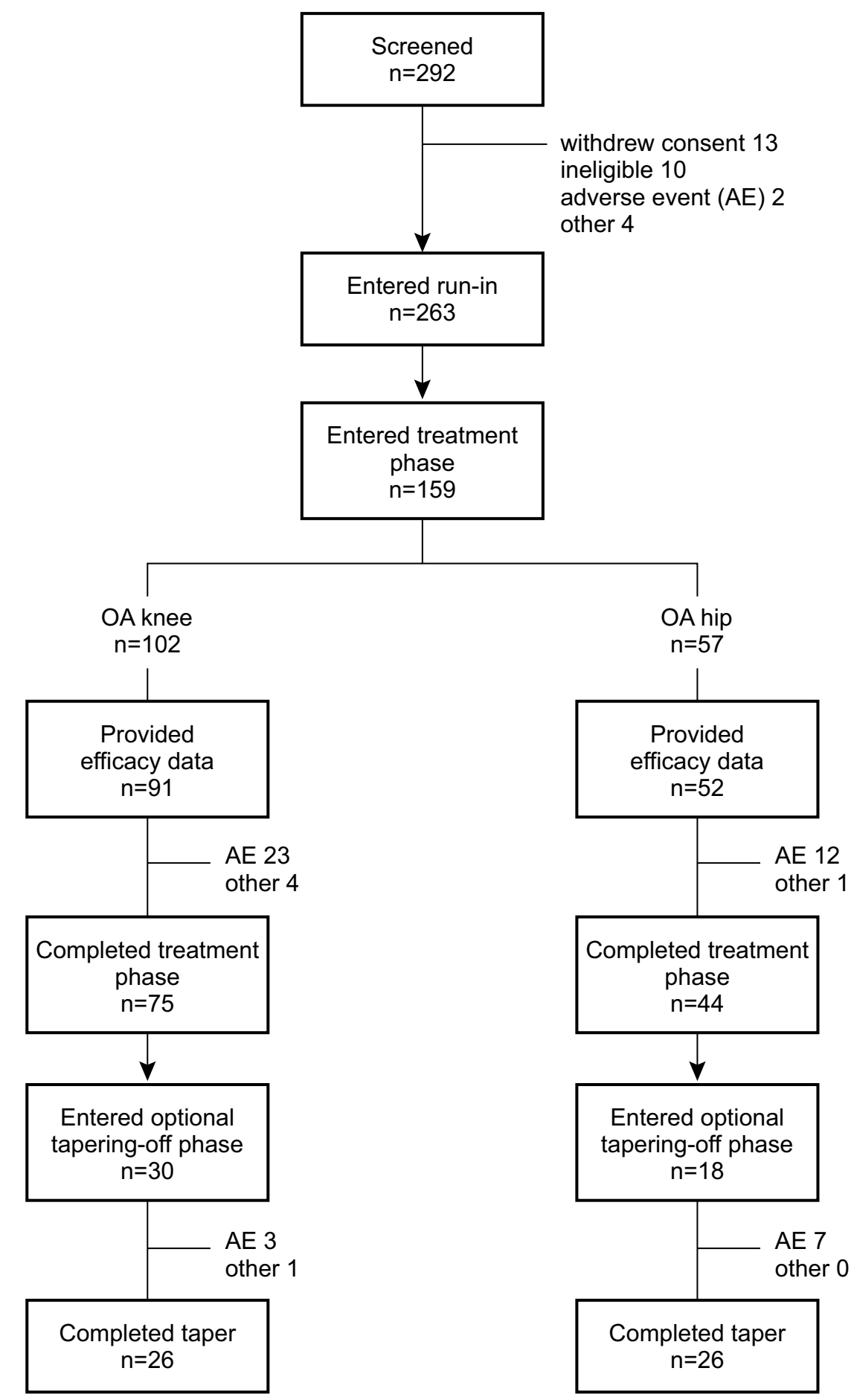

Figure I

Patient disposition. 


\section{Treatment assessment}

In their assessment of treatment (total $\mathrm{n}=125$, OA knee 82 , OA hip 43), 63\% of patients rated TDF positively with respect to pain control and $84 \%$ would recommend TDF for their type of pain. Most patients were satisfied with its convenience of use (93\% thought it easy/extremely easy to use; $85 \%$ were very/somewhat pleased by the way it's used), and 53\% considered side effects were not an issue. In general, there was a difference of less than $10 \%$ between the OA knee and OA hip groups. In assessing how they had felt over the past week, the percentage of all patients who answered good or very good increased during the study from $7 \%(10 / 142)$ during the run-in period to $32 \%(31 / 97)$ in week 4 , and their scores at all time points were significantly better than before treatment $(\mathrm{p}<$ $0.001)$. By the end of the study, help with basic activities was required by only $28 \%$ of patients, with $49 \%$ relying less on their helper.

\section{Quality of life}

For the 122 patients who completed the SF-36 quality of life questionnaire, there were statistically significant improvements in all domains from baseline to endpoint, including overall physical health $(\mathrm{p}<0.001)$ and mental health $(\mathrm{p}<0.05)$ (Table 3$)$. Despite optimization of previous treatment, quality of life scores were low at baseline, with the patients' underlying osteoarthritis particularly

Table 2: Pain control assessments (number of patients and percentage in each category)

\begin{tabular}{lccc}
\hline & Screening N (\%) & Baseline N (\%) & Endpoint N (\%) \\
\hline Very poor & $27(19)$ & $36(25)$ & $4(3)$ \\
Poor & $110(77)$ & $68(48)$ & $21(14)$ \\
Moderate & $5(3)$ & $36(25)$ & $53(37)$ \\
Good & $\mathrm{I}(1)$ & $2(2)$ & $54(38)$ \\
Excellent & 0 & 0 & $11(8)$ \\
\hline
\end{tabular}

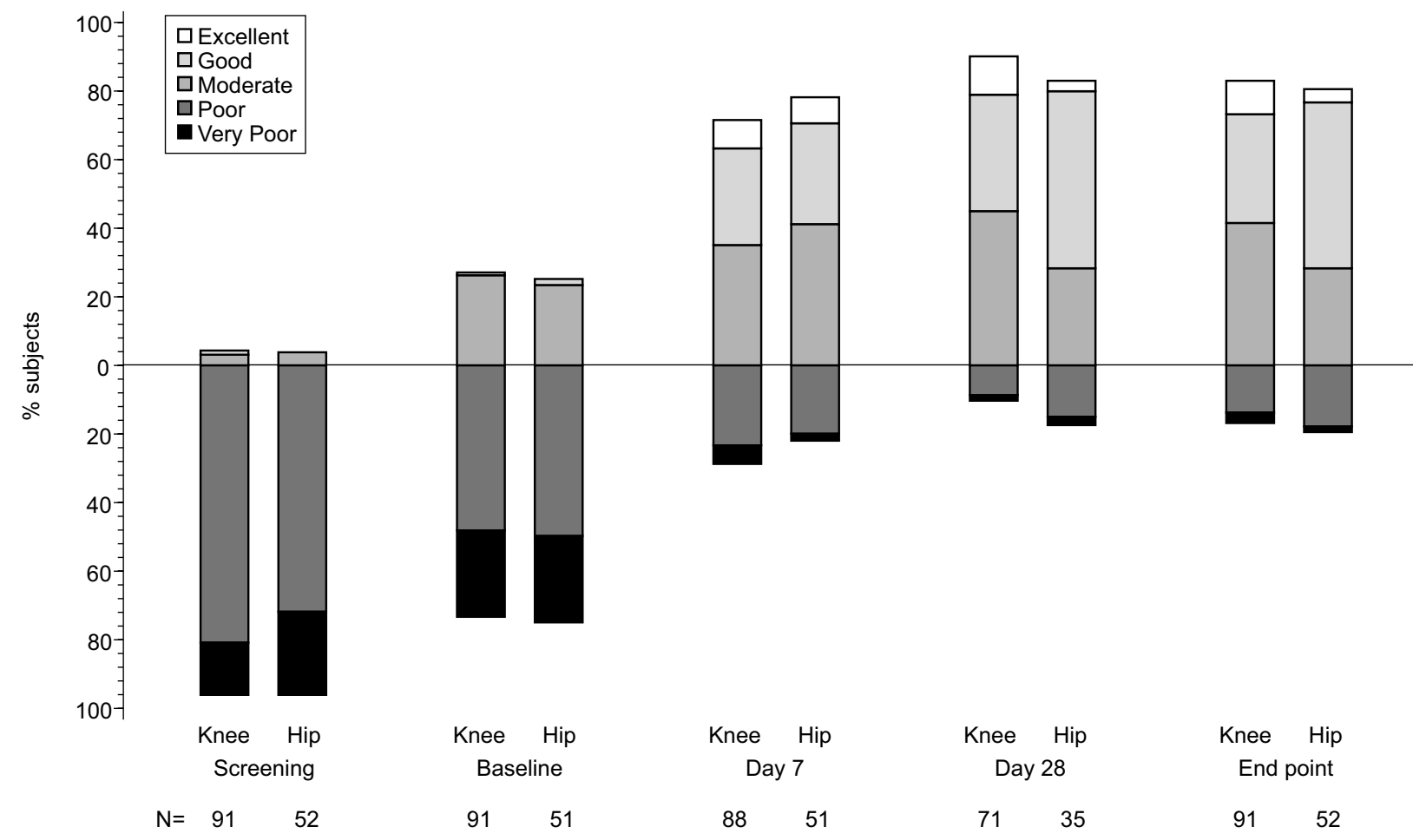

Pain relief

\section{Figure 2}

Pain control assessment. 
Table 3: Quality of life (SF-36 scores)

\begin{tabular}{|c|c|c|c|c|}
\hline Domain & $\mathbf{n}$ & $\begin{array}{c}\text { Score at baseline (Mean } \\
\pm \mathrm{SE})\end{array}$ & $\begin{array}{c}\text { Mean change between } \\
\text { baseline and endpoint } \\
(95 \% \mathrm{Cl})\end{array}$ & $p$-value \\
\hline \multicolumn{5}{|l|}{ Physical functioning } \\
\hline Physical functioning & 120 & $30.0 \pm 2.15$ & $4.0(-0.09,7.99)$ & $<0.05$ \\
\hline Role physical & 119 & $11.8 \pm 2.38$ & $15.8(8.89,22.62)$ & $<0.001$ \\
\hline Bodily pain & 122 & $23.7 \pm 1.54$ & I7.I (13.21, 20.97) & $<0.001$ \\
\hline General health & 119 & $44.1 \pm 2.09$ & $3.6(0.09,7.05)$ & $<0.05$ \\
\hline \multicolumn{5}{|l|}{ Mental health } \\
\hline Vitality & 119 & $34.6 \pm 1.74$ & $6.3(3.24,9.45)$ & $<0.001$ \\
\hline Social functioning & 122 & $50.3 \pm 2.55$ & $7.992 .99,12.79$ ( & $<0.05$ \\
\hline Role emotional & 117 & $34.9 \pm 3.97$ & $10.8(1.85,19.81)$ & $<0.05$ \\
\hline Mental health & 118 & $53.0 \pm 2.06$ & $4.7(1.28,8.03)$ & $<0.05$ \\
\hline \multicolumn{5}{|l|}{ Summary measures } \\
\hline Physical health & 111 & $27.0 \pm 0.69$ & $4.1(2.73,5.54)$ & $<0.001$ \\
\hline Mental health & III & $41.7 \pm 1.17$ & $2.5(0.46,4.64)$ & $<0.05$ \\
\hline
\end{tabular}

Table 4: WOMAC scores (mean normalised score $\pm \mathrm{SE}$, all changes from baseline are statistically significant, $\mathrm{p}<0.00 \mathrm{I}$ in all cases)

\begin{tabular}{lccc}
\hline & Baseline & Endpoint & $\begin{array}{c}\text { Change from baseline to } \\
\text { endpoint }(95 \% \mathrm{Cl})\end{array}$ \\
\hline Pain & $6.3 \pm 0.15$ & $4.6 \pm 0.21$ & $-1.7(-2.05,-1.26)$ \\
Stiffness & $6.1 \pm 0.19$ & $4.7 \pm 0.23$ & $-1.4(-1.89,-1.0)$ \\
Physical functioning & $6.6 \pm 0.15$ & $5.1 \pm 0.19$ & $-1.5(-1.83,-1.14)$ \\
Overall & $19.0 \pm 0.4$ & $14.3 \pm 0.57$ & $-4.7(-5.69,-3.62)$ \\
\hline
\end{tabular}

affecting role physical and bodily pain. It was in these two areas that patients showed greatest absolute improvement with TDF treatment.

\section{WOMAC}

The mean score for all 24 questions from the three summary parameters (pain, stiffness and physical functioning) improved significantly from baseline to endpoint for all groups (total population $\mathrm{p}<0.001$, knee $\mathrm{p}<$ 0.001 , and hip $\mathrm{p}<0.05$ for all questions) (Table 4 ). The percentage of patients in the combined group and in the knee sub-group who reported no pain, stiffness or physical difficulties increased for all items. A similar increase occurred in the OA hip group except for 'stiffness after first awakening', 'rising from bed' and 'getting in/out of the bath' which showed little change.

The majority of participants showed an improvement in score for the three summary measurements of pain, stiff- ness and physical functioning, and for the overall WOMAC score. Mean overall WOMAC score improved significantly $(\mathrm{p}<0.001)$ from baseline to endpoint (Table 4). In addition, the change from baseline to endpoint in score for 'pain right now' (from the pain assessment questionnaire) showed a weak positive correlation with the change in overall WOMAC score (Spearman correlation coefficient: non-normalized 0.344 and normalized $0.384)$.

\section{Evaluation of safety}

Adverse events occurring during the treatment phase and tapering off phase were those associated with strong opioid treatment (Table 5). Adverse events were reported by $6 \%(9 / 159)$ of patients during the run-in period, $65 \%$ $(68 / 104)$ of patients during the treatment period and $25 \%$ (12/48) during the optional tapering off period. The study medication was permanently stopped in 25\% (39) of cases, particularly because of nausea (53\%), vomiting 
Table 5: Adverse events (AEs) reported during the treatment phase and tapering off phase by $>5 \%$ of participants

\begin{tabular}{lc}
\hline Preferred term & Total AEs n (\%) \\
\hline & Treatment phase $(\mathrm{N}=104)$ \\
Nausea & $51(32 \%)$ \\
Vomiting & $41(26 \%)$ \\
Somnolence & $25(16 \%)$ \\
Dizziness & $14(9 \%)$ \\
Constipation & $10(6 \%)$ \\
Asthenia & $9(6 \%)$ \\
Pruritus & $8(5 \%)$ \\
& $5(10 \%)$ \\
Nausea & Tapering off phase $(\mathrm{N}=42)$ \\
Vomiting & $3(6 \%)$ \\
\hline
\end{tabular}

$(47 \%)$ and dizziness (18\%). (No falls or fractures were reported.) Withdrawal syndrome was reported in two cases (OA hip) during tapering off - one was mild, the other moderate, and both resolved without specific treatment. No deaths occurred. Two patients reported at least one serious adverse event during the treatment phase (severe asthenia and anorexia in one case, bronchitis in the other) and one experienced a serious adverse event during the tapering-off phase (hospitalization due to chest pain and arrythmia), but these were considered unrelated to the study drug. There were no clinically significant changes in vital signs during the study.

\section{Discussion}

The study was intended to evaluate the utility of TDF under routine conditions and to investigate different practical issues, such as the usefulness of a test-period and use of concomitant antiemetic treatment during the first weeks. It was not designed as a primary efficacy study, since the efficacy of TDF in providing pain relief has already been demonstrated [14-18]. For this reason it was considered unethical to include a placebo control group.

A one-month test period [10] is sufficient to show a role for TDF in the treatment of pain caused by OA of the knee or hip that is not adequately controlled by NSAIDs, Cox2 inhibitors, paracetamol or weak opioids at optimal doses. Findings support previous conclusions that OAinduced pain can be successfully treated with TDF, and that this may result in improved functioning [22]. Not all patients with OA of the knee or hip receive adequate doses of their current non-opioid analgesia because pain control can be improved in some when doses of these medications are optimized. Undertreatment of OA-related pain has been reported previously [24]. Adequate pain control was achieved for most patients after one week of TDF, at the starting dose of $25 \mu \mathrm{g} / \mathrm{hr}$ in over half of all patients, and relief was maintained over the treatment period. Pain was controlled in $88 \%$ of patients after one month with nearly $40 \%$ reporting mild or no pain.

A few patients started treatment with TDF, despite adequate pain control during the run-in phase. These patients were considered to be protocol violators. However, their inclusion provided the opportunity to determine whether TDF could give additional benefits to these patients above those already gained by their current medications. About half of these patients achieved further pain reduction while treated with TDF.

Over the treatment period, the numbers of all patients using other analgesics, especially NSAIDs decreased substantially (from $45 \%$ to $19 \%$ ). This may be beneficial in reducing side effects such as gastrointestinal bleeding. Nearly all patients taking weak opioids took them on the first day of treatment only when serum levels of fentanyl had not yet achieved steady state. Paracetamol may be useful for breakthrough pain in some patients but need for rescue medication should be evaluated on an individual basis. Metoclopramide did not appear to prevent nausea or vomiting.

Treatment was considered favourable in terms of efficacy, side effects and convenience. A preference for treatment with TDF over sustained release morphine has previously been shown by patients with chronic non-cancer pain [16].

The general health measure, SF-36, indicated that control of pain significantly improved both physical and mental components of quality of life. The improvement in mental health may be related to improved functioning which permits greater social activity. In spite of similar pain control, patients with OA of the hip appeared to have slightly more difficulty with stiffness and general mobility than those with OA of the knee, such as getting in/out of the bath or bed, probably due to the location and function of these joints.

WOMAC is a reliable, valid and responsive multidimensional, self-administrated outcome measure designed specifically to evaluate patients with OA of the knee or hip [27]. Overall pain, stiffness and function significantly improved after one month of TDF treatment for both OA knee and OA hip patients, with improvements in nearly all items of WOMAC summary categories. Quality of life would be expected to be improved with pain relief, although significant pain relief would not necessarily be associated with reduced stiffness and increased physical function [29]. Increased functioning was also indicated by the fact that half of all patients required less help with 
daily activities of living. Return of a full range of motion is unlikely to occur when marked structural damage of the joint has occurred.

Overall, the spectrum of reported side effects was consistent with those commonly associated with opioid therapy and with previous experience with TDF. Constipation was not a problem for this patient population as with other patients receiving TDF for non-cancer pain or cancer pain $[16,29,30]$, and tolerance to other side effects is likely to develop with continued treatment [31]. In addition to nausea and vomiting, patients should be warned of the possibility of dizziness when starting strong opioid treatment. This is especially important for the elderly OA population in order to prevent falls. In the present study, $9 \%$ of patients reported dizziness.

As might be expected in an elderly population, many patients had co-existing diseases. For example, 59\% had cardiovascular disease on entry, which is of particular interest given the concern about the cardiovascular safety of some Cox-2 inhibitors which, since this trial was undertaken, has led to the withdrawal of rofecoxib.

This trial demonstrates that patients with OA of the knee or hip continue to experience pain even at optimal doses of their non-opioid treatment, providing a major reason why patients and clinicians alike are often dissatisfied with current therapies. The study also shows that patients with OA benefit from additional pain control provided by TDF [32]. Clinicians are beginning to accept that strong opioids are well tolerated and effective and should be made available when non-opioids have failed to control pain [33]. Our findings support this reasoning and suggest that opioids should be made more widely available where appropriate.

\section{Statement of competing interest}

Dr Richarz is an employee of Janssen-Cilag and the other authors have both received research funding from Janssen-Cilag, which funded this study.

\section{Authors' contributions}

UR designed the study and coordinated it. XLL and KP recruited patients to the study and contributed to the interpretation of the findings. All authors contributed to developing the manuscript for publication.

\section{Acknowledgements}

This study was funded by Janssen-Cilag. We would like to acknowledge the FEN-INT-30 Study Group for their participation in this trial and to thank Dr Susan Libretto for help in preparing the manuscript.

\section{References}

I. Lawrence JS, Bremner JM, Bier F: Osteoarthritis, prevalence in the population and relationship between symptoms and $X$ ray changes. Ann Rheum Dis 1966, 25:I-24.

2. Arthritis Research Campaign: Factfile - arthritis at a glance. 2003 [http://http.//www.arc.org.uk].

3. Creamer P, Flores R, Hochberg MC: Management of osteoarthritis in older adults. Clin Geriatr Med 1998, I4:435-454.

4. Pham T, van der Heijde D, Lassere M, Altman RD, Anderson JJ, Bellamy N, Hochberg M, Simon L, Strand V, Woodworth T, Dougados M: Outcome variables for osteoarthritis clinical trials: The OMERACT-OARSI set of responder criteria. J Rheumatol 2003, 30:1648-1654.

5. Anon: Recommendations of the medical management of osteoarthritis of the hip and knee: 2000 update. In Arthritis Rheum Volume 43. American College of Rheumatology Subcommittee on Osteoarthritis; 2000:1905-1915.

6. Roth SH, Reder RF: The role of opioids in the treatment of osteoarthritis. Resident Staff Physician 1998, 44:31-36.

7. Wolheim FA: Current pharmacological treatment of osteroarthritis. Drugs 1996, 52(supp 3):27-38.

8. Pinals RS: Mechanism of joint destruction, pain and disability in osteoarthritis. Drugs 1996, 52(Supp 3): | 4-20.

9. American Pain Society: Guideline for the management of pain in osteoarthritis, rheumatoid arthritis, and juvenile chronic arthritis. 2002 [http://www.ampainsoc.org].

10. Kalso E, Allan L, Dellemijn PLI, Faura CC, Ilias WK, Jensen TS, Perrot $\mathrm{S}$, Plaghki LH, Zenz M: Recommendations for using opioids in chronic non-cancer pain. Eur J Pain 2003, 7:38I-386.

II. Schug SA, Merry AF, Acland RH: Treatment principles for the use of opioids in pain of nonmalignant origin. Drugs 1991, 42:228-239.

12. Graziotti PJ, Goucke CR: The use of oral opioids in patients with chronic non-cancer pain. Management strategies. Med J Aust 1997, 167:30-34.

13. Anon: The use of opioids for the treatment of chronic pain: a consensus statement from the American Academy of Pain Medicine and the American Pain Society. Pain Forum 1997, 6:77-79.

14. Jeal W, Benfield P: Transdermal fentanyl: a review of its pharmacological properties and therapeutic efficacy in pain control. Drugs 1997, 53:109-138.

15. Simpson RK, Edmondson EA, Constant CF, Collier C: Transdermal fentanyl as treatment for chronic low back pain. J Pain Symptom Manage 1997, 14:218-224.

16. Allan L, Hays H, Jensen NH, de Waroux BL, Bolt M, Donald R, Kalso $E$ : Randomised crossover trial of transdermal fentanyl and sustained release oral morphine for treating chronic noncancer pain. BMJ 200I, 322: I| I54-II 58.

17. Milligan K, Lanteri-Minet M, Borchert K, Helmers H, Donald R, Kress $\mathrm{HG}$, Adriaensen H, Moulin D, Jarvimaki V, Haazen L: Evaluation of long term efficacy and safety of transdermal fentanyl in the treatment of chronic non-cancer pain. J Pain 200I, 2:197-204.

18. Ringe JD, Faber $H$, Bock $O$, Valentine S, Felsenberg D, Pfeifer M, Minne HW, Schwalen S: Transdermal fentanyl for the treatment of back pain caused by vertebral osteoporosis. Rheumatol Int 2002, 22:199-203.

19. Peloso PM, Bellamy N, Bensen W, Thompson GT, Harsanyi Z, Babul $N$, Darke AC: Double blind randomized placebo control trial of controlled release codeine in the treatment of osteoarthritis of the knee or hip. J Rheumatol 2000, 27:764-77I.

20. Caldwell JR, Hale ME, Boyd RE, Hague JM, Iwam T, Shi M, Lacouture PG: Treatment of osteoarthritis pain with controlled release oxycodone or fixed combination oxycodone plus acetaminophen added to nonsteroidal anti-inflammatory drugs: a double blind, randomized, multicentre, placebo controlled trial. J Rheumatol 1999, 26:862-869.

21. Caldwell JR, Rapoport RJ, Davis JC, Offenberg HL, Marker HW, Roth $\mathrm{SH}$, Yuan W, Eliot L, Babul N, Lynch PM: Efficacy and safety of a once-daily morphine formulation in chronic, moderate-tosevere osteoarthritis pain: results from a randomized, placebo-controlled, double-blind trial and an open-label extension trial. J Pain Symptom Manage 2002, 23:278-29I.

22. Theodoridis T, Waap I, Schwalen S, Kramer J: Fentanyl in the treatment of pain caused by arthrosis. Z Orthop 2002, 1 40:2 17-222. 
23. Bierma-Zeinstra S, Bohnen A, Ginai A, Prins A, Verhaar J: Validity of the American College of Rheumatology criteria for diagnosing hip osteoarthritis in primary care research. I Rheumatol 1999, 26: I| 29-II33.

24. Bellamy N, Bradley LA: Workshop on chronic pain, pain control, and patient outcomes in rheumatoid arthritis and osteoarthritis. Arthritis Rheum 1996, 39:357-362.

25. Cleeland CS, Ryan KM: Pain assessment: global use of the Brief Pain Inventory. Ann Acad Med Singapore 1994, 23:129-I38.

26. Ware JE, Sherbourne CD: The MOS 36-item Short-Form Health Survey (SF-36). Med Care 1992, 30:473-483.

27. Bellamy N, Buchanan WW, Goldsmith CH, Campbell J, Stitt LW: Validation Study of WOMAC: A health status instrument for measuring clinically important patient relevant outcomes to antirheumatic drug therapy in patients with osteoarthritis of the knee or hip. J Rheumatology 1988, 15:1833-1840.

28. Gillings $D$, Koch $G$ : The application of the principle of intention-to-treat to the analysis of clinical trials. Drug Inform J I99I, 25:4II-424.

29. Allan L, Simpson K, Slappendel R: Transdermal fentanyl versus sustained release oral morphine in strong-opioid naïve patients with chronic low back pain. Spine 2005 in press.

30. Ahmedzai S, Brooks D: Transdermal fentanyl versus sustained release oral morphine in cancer pain: preference, efficacy and quality of life. J Pain Symptom Manage 1997, 13:254-26I.

31. Dellemijn PLI, van Duijn H, Vanneste JAL: Prolonged treatment with transdermal fentanyl in neuropathic pain. J Pain Symptom Manage 1998, 16:220-229.

32. Knott L: Treating osteoarthritis in practice - the TOP study. Curr Med Res Opin 2000, 16:147-152.

33. Portenoy RK: Current pharmacotherapy of chronic pain. J Pain Symptom Manage 2000, 19:SI6-S20.

\section{Pre-publication history}

The pre-publication history for this paper can be accessed here:

http://www.biomedcentral.com/1471-2474/6/31/prepub

Publish with Bio Med Central and every scientist can read your work free of charge

"BioMed Central will be the most significant development for disseminating the results of biomedical research in our lifetime. "

Sir Paul Nurse, Cancer Research UK

Your research papers will be:

- available free of charge to the entire biomedical community

- peer reviewed and published immediately upon acceptance

- cited in PubMed and archived on PubMed Central

- yours - you keep the copyright

Submit your manuscript here:

http://www.biomedcentral.com/info/publishing_adv.asp
BiolMedcentral 